\title{
Binocular Disparity Tuning and Visual-Vestibular Congruency of Multisensory Neurons in Macaque Parietal Cortex
}

\author{
Yun Yang, ${ }^{1 \star}$ Sheng Liu, ${ }^{1 \star}$ Syed A. Chowdhury, ${ }^{1}$ Gregory C. DeAngelis, ${ }^{2}$ and Dora E. Angelaki ${ }^{3}$ \\ ${ }^{1}$ Department of Anatomy and Neurobiology, Washington University School of Medicine, St. Louis, Missouri 63110, 2 Department of Brain and Cognitive \\ Sciences, Center for Visual Science, University of Rochester, Rochester, New York 14627, and ${ }^{3}$ Department of Neuroscience, Baylor College of Medicine, \\ Houston Texas 77030
}

\begin{abstract}
Many neurons in the dorsal medial superior temporal (MSTd) and ventral intraparietal (VIP) areas of the macaque brain are multisensory, responding to both optic flow and vestibular cues to self-motion. The heading tuning of visual and vestibular responses can be either congruent or opposite, but only congruent cells have been implicated in cue integration for heading perception. Because of the geometric properties of motion parallax, however, both congruent and opposite cells could be involved in coding self-motion when observers fixate a world-fixed target during translation, if congruent cells prefer near disparities and opposite cells prefer far disparities. We characterized the binocular disparity selectivity and heading tuning of MSTd and VIP cells using random-dot stimuli. Most (70\%) MSTd neurons were disparity selective with monotonic tuning, and there was no consistent relationship between depth preference and congruency of visual and vestibular heading tuning. One-third of disparity-selective MSTd cells reversed their depth preference for opposite directions of motion [direction-dependent disparity tuning (DDD)], but most of these cells were unisensory with no tuning for vestibular stimuli. Inconsistent with previous reports, the direction preferences of most DDD neurons do not reverse with disparity. By comparison to MSTd, VIP contains fewer disparity-selective neurons (41\%) and very few DDD cells. On average, VIP neurons also preferred higher speeds and nearer disparities than MSTd cells. Our findings are inconsistent with the hypothesis that visual/vestibular congruency is linked to depth preference, and also suggest that DDD cells are not involved in multisensory integration for heading perception.
\end{abstract}

\section{Introduction}

Patterns of image motion across the retina ("optic flow") provide powerful cues to heading (Gibson, 1950; Warren, 2003). Independent information about translation of the head arises from the otolith organs of the vestibular system (Angelaki and Cullen, 2008). Visual and vestibular cues naturally arise together during self-motion, and neurons sensitive to the convergence of these cues have been described in areas MSTd (Duffy, 1998; Bremmer et al., 1999; Page and Duffy, 2003; Gu et al., 2006, 2008, 2010) and VIP (Bremmer et al., 2002; Schlack et al., 2002; Chen et al., 2011b), as well as in the visual posterior sylvian area (Chen et al., 2011a). Two types of multisensory neurons have been found in these areas (Gu et al., 2006): (1) neurons with congruent visual and vestibular heading preferences and (2) neurons with opposite visual/vestibular heading preferences, which should never be maximally activated when an observer translates through a static environment without moving their eyes. Whereas congruent cells

Received Aug. 5, 2011; revised Sept. 26, 2011; accepted 0ct. 19, 2011.

Author contributions: Y.Y., S.L., G.C.D., and D.E.A. designed research; Y.Y., S.L., and S.A.C. performed research;

Y.Y., S.L., and S.A.C. analyzed data; Y.Y., S.L., G.C.D., and D.E.A. wrote the paper.

The work was supported by NIH Grants EY019087 (D.E.A.) and EY016178 (G.C.D.).

*Y.Y. and S.L. contributed equally to this work.

Correspondence should be addressed to Dr. Dora E. Angelaki, Department of Neuroscience, Baylor College of Medicine, One Baylor Plaza, Room S740, MS: BCM295, Houston, TX 77030. E-mail: angelaki@bcm.edu.

DOI:10.1523/JNEUROSCI.4032-11.2011

Copyright $\odot 2011$ the authors $\quad 0270-6474 / 11 / 3017905-12 \$ 15.00 / 0$ show improved sensitivity and correlations with perceptual decisions during visual-vestibular cue integration, neither is true for opposite neurons (Gu et al., 2008).

What, then, might be the function of opposite cells? One possibility is that they play a role in coding self-motion under viewing conditions in which a particular heading produces two possible directions of retinal image motion. When a translating observer fixates a world-fixed target, near and far objects (relative to fixation) move in opposite directions on the retina (Fig. $1 A$ ). However, the identical observer translation during fixation of a head-fixed target results in the same direction of retinal image motion for near and far objects (Fig. $1 B$ ). In our previous studies, congruency of visual and vestibular heading tuning was measured during fixation of a head-fixed target (Fig. $1 B$ ), and depth preferences of individual cells were not known. If opposite cells preferred far disparities and congruent cells preferred near disparities, then both cell types would represent physically consistent optic flow and inertial motion when an observer fixates a world-fixed target during self-motion. While it is known that most MSTd neurons are selective for binocular disparity (Roy et al., 1992; Takemura et al., 2001), the relationship between depth preference and visual-vestibular congruency is unknown.

An additional complexity is that some MSTd cells reverse their disparity preference for opposite directions of motion (DDD cells) (Roy and Wurtz, 1990; Roy et al., 1992). Such neurons may play an important role in representing self-motion during fixa- 
A
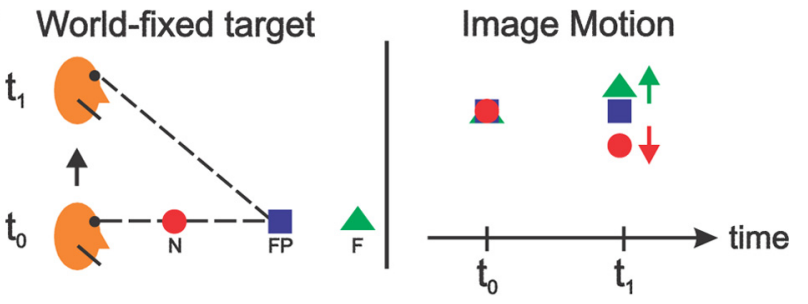

B

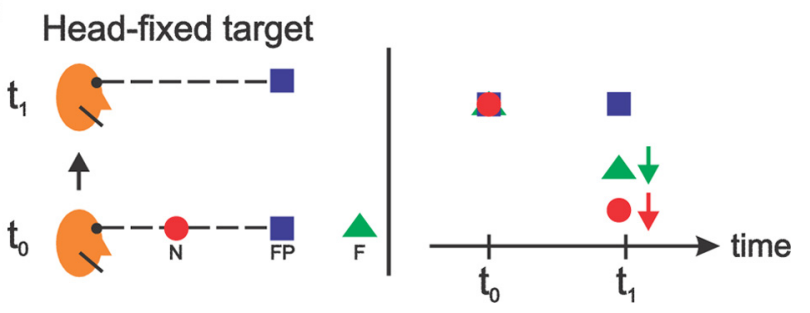

Figure 1. Viewing geometry and the relationships between depth and retinal velocity. $\boldsymbol{A}$, When a subject fixates a world-fixed target while translating upward, near objects move downward on the display screen, whereas far objects move upward. $\boldsymbol{B}$. When the same selftranslation occurs while fixating a head-fixed target, both near and far objects move downward in the display.

tion on a world-fixed target (Roy et al., 1992). However, the relationship between the DDD property and visual-vestibular convergence is not known, nor is it clear whether DDD cells even receive vestibular input.

We investigated whether visual-vestibular congruency is related to the disparity preferences of MSTd and VIP neurons, as well as the DDD property. We did not observe a correlation between visual-vestibular congruency and depth selectivity, suggesting that the existence of opposite cells is not simply related to viewing geometry but rather serves other functional roles such as dissociation of self-motion and object motion.

\section{Materials and Methods}

\section{Animal preparation}

Four male monkeys (Macaca mulatta) were used for neurophysiological recordings. Our general procedures have been reported in detail elsewhere (Gu et al., 2006), so they will be described only briefly here. Under sterile conditions, monkeys were chronically implanted with a ring-type device for head stabilization. Scleral coils were implanted in both eyes for monitoring eye position, including both version and vergence (Robinson, 1963; Judge et al., 1980). A bilateral recording grid was positioned in the horizontal plane and extended from the midline outward to the areas overlying MSTd and VIP bilaterally. The recording grid contained staggered rows of holes $(0.8 \mathrm{~mm}$ spacing $)$ and was stereotaxically secured inside the head cap using dental acrylic. Vertical microelectrode penetrations were made via transdural guide tubes inserted through the grid holes. Behavioral training was performed using standard operant conditioning techniques. All animal surgeries were approved by the Institutional Animal Care and Use Committee at Washington University and were in accordance with National Institutes of Health guidelines.

\section{Vestibular and visual stimuli}

Heading stimuli. Monkeys sat comfortably in a primate chair mounted on top of a six degrees of freedom motion platform (MOOG 6DOF2000E; Moog). Visual stimuli were delivered using a three-chip DLP projector (Mirage 2000 or Mirage S+2k; Christie Digital Systems), which rear-projected images onto a tangent screen that was viewed by the monkey from a distance of $30 \mathrm{~cm}$ (subtending $\sim 90 \times 90^{\circ}$ of visual angle). The entire projection apparatus was mounted on top of the motion platform such that visual stimuli could be controlled independently of the movement trajectory. The tangent screen was mounted at the front of the field coil frame, with the sides, top, and bottom of the frame covered with a black enclosure. Thus, only visual motion projected onto the display screen was visible to the animal.

The visual display had a pixel resolution of $1280 \times 1024$ and 32-bit color depth, and was updated at the same rate as the movement trajectory $(60 \mathrm{~Hz})$. Optic flow stimuli were generated by an OpenGL accelerator board (nVidia Quadro FX 3000G) housed in a dedicated dual-processor PC. Visual stimuli were plotted with subpixel accuracy using hardware anti-aliasing under OpenGL, allowing presentation of smooth motion trajectories and binocular disparities much smaller than the distance between pixels.

Visual stimuli in the heading tuning protocol depicted movement of the observer through a 3D cloud of "stars" $(100 \mathrm{~cm}$ wide, $100 \mathrm{~cm}$ tall, and $40 \mathrm{~cm}$ deep). Star density was $0.01 / \mathrm{cm}^{3}$, with each star being a $0.15 \times$ $0.15 \mathrm{~cm}$ triangle. Approximately 1500 stars were visible at any time within the field of view of the screen. The display screen was located in the center of the star field before stimulus onset and remained well within the depth of the star field throughout the motion trajectory. Stereoscopic images were displayed as red/green anaglyphs and were viewed through Kodak Wratten filters (\#29, \#61) that were mounted on custom-made goggles.

Disparity tuning stimuli. For measurements of disparity selectivity and $2 \mathrm{D}$ direction tuning (in the frontoparallel plane), full-field $\left(90 \times 90^{\circ}\right)$ random-dot stimuli were generated by OpenGL in a $2 \mathrm{D}$ orthographic rendering context. These stimuli were also viewed as red/green anaglyphs. Intensities of the red and green dots were adjusted to have approximately equal luminance, as measured through the corresponding red and green filters with a photometer. Dots moved coherently, and their trajectories wrapped around when the edge of the aperture was reached.

Monkeys were required to maintain fixation on a central, head-fixed target throughout the duration of the stimulus to obtain a liquid reward. Positions of both eyes were monitored using scleral coils. Monkeys were trained to maintain version within a $2^{\circ}$ square electronic window, whereas vergence angle was enforced to lie within $\pm 0.5^{\circ}$ of the plane of fixation. Maintenance of the correct vergence angle is important such that the desired binocular disparities were rendered on the retinae. We found a weak but significant dependence of vergence angle on stimulus disparity for each of the monkeys (Monkey F, slope $0.005 ; 95 \%$ CI, $[0.003$ 0.006]; $p<0.01$; Monkey O, slope, 0.004; 95\% CI, [0.003 0.006]; $p<$ 0.001; Monkey P, slope, 0.009; 95\% CI, [0.006 0.012]; $p<0.001$; Monkey $\mathrm{X}$, slope, $0.007 ; 95 \% \mathrm{CI}$, [0.005 0.008]; $p<0.01$; linear regression). Although these effects were significant (due to having large amounts of data), they amount to less than a $1 \%$ change in vergence angle per degree of stimulus disparity, and thus would have a negligible impact on the measured disparity tuning of MSTd/VIP neurons.

\section{Electrophysiological recordings}

Tungsten microelectrodes (tip diameter, $3 \mu \mathrm{m}$; impedance, $1-2 \mathrm{M} \Omega$ at 1 $\mathrm{kHz}$; Frederick Haer) were advanced into the cortex through a transdural guide tube, using a hydraulic microdrive (Frederick Haer). Behavioral control and data acquisition were accomplished using a commercially available software package (TEMPO; Reflective Computing). Action potentials were amplified, filtered ( 250 to $8000 \mathrm{~Hz}$ ), and sorted online using the Plexon data acquisition system (Plexon). The times of occurrence of action potentials and all behavioral events were recorded with $1 \mathrm{~ms}$ resolution. Eye-movement traces were sampled at a rate of $200 \mathrm{~Hz}$. In addition, raw neural signals were digitized at a rate of $25 \mathrm{kHz}$ using a CED Power 1401 data acquisition system (Cambridge Electronic Design) along with Spike2 software. These raw data were stored to disk for off-line spike sorting and additional analyses.

Areas MSTd and VIP were first located in stereotaxic coordinates with the aid of magnetic resonance imaging (MRI) scans (for details, see Gu et al., 2006; Chen et al., 2011b). These areas were then functionally localized based on patterns of gray and white matter transitions along electrode penetrations and by mapping of physiological response properties. Our procedure for identifying area MSTd was described in detail previously (Gu et al., 2006, 2008; Takahashi et al., 2007). To identify the VIP, with the aid of MRI scans, we performed electrode penetrations within a large region of cortex around the expected location of the intraparietal cortex 
(Chen et al., 2011b). At each anterior/posterior location, we located the medial tip of the intraparietal sulcus and moved laterally until directionally selective visual responses were absent in multiunit activity. For most VIP neurons, we mapped the receptive field (RF) by manually controlling a patch of drifting random dots while observing a map of instantaneous firing rates on a custom graphical interface. VIP RFs were generally contralateral but often extended into the ipsilateral visual field and included the fovea. In addition, VIP neurons were typically activated only by moderately large visual stimuli $\left(>10 \times 10^{\circ}\right)$, with little response evoked from smaller patches.

\section{Experimental protocols}

Heading tuning protocol. Upon isolating the action potential of a neuron, its visual and vestibular heading tuning were characterized. This protocol was used to classify neurons as unisensory (visual only or vestibular only) or multisensory, and was also used to assess the congruency of visual and vestibular tuning. Within the same block of trials, two stimulus conditions were interleaved: a "vestibular" condition in which the monkey was translated by the motion platform in the absence of optic flow (the screen was blank, except for a head-centered fixation point), and a "visual" condition in which the motion platform was stationary while optic flow simulated movement through a 3D cloud of stars (for details, see Gu et al., 2006). Each trial lasted $2 \mathrm{~s}$, although most of the movement occurred within the middle $1 \mathrm{~s}$. The stimulus trajectory had a Gaussian velocity profile and a corresponding biphasic acceleration profile. The motion amplitude was $13 \mathrm{~cm}$ (total displacement), with a peak acceleration of $\sim 0.1 \mathrm{~g}\left(\sim 0.98 \mathrm{~m} / \mathrm{s}^{2}\right)$ and a peak velocity of $\sim 30 \mathrm{~cm} / \mathrm{s}$. To assess the spontaneous activity of each neuron, a null condition without platform motion or optic flow was also interleaved. For each trial, the animal was required to fixate a central target $\left(0.2^{\circ}\right.$ in diameter $)$ for $200 \mathrm{~ms}$ before onset of the motion stimulus, and the animal was rewarded at the end of each trial for maintaining fixation throughout the stimulus presentation.

For $\sim 40 \%$ of MSTd neurons and $80 \%$ of VIP neurons, the heading tuning protocol consisted of stimuli presented along 26 directions in 3D space, corresponding to all combinations of azimuth and elevation angles in increments of $45^{\circ}$ (265 trials for five repetitions of each distinct stimulus). For the other $60 \%$ of MSTd neurons and $20 \%$ of VIP neurons, a reduced heading tuning protocol was used, consisting of eight directions of translation in the horizontal plane (azimuth angles of $0,45,90$, $135,180,225,270$, and $315^{\circ}$ ) and two vertical directions (elevation angles of -90 and $90^{\circ}$ ). With this reduced experimental protocol, 100 trials were required to complete five repetitions of all stimuli. Because some VIP neurons did not respond significantly to optic flow stimuli, whereas essentially all MSTd neurons did (Gu et al., 2006; Chen et al., 2011b), the significance of visual heading tuning was assessed on-line (one-way ANOVA), and only cells with significant visual heading tuning $(p<$ 0.05 ) were tested further.

Disparity-direction tuning protocol. In this protocol, we tested neurons with all combinations of eight visual motion directions in the frontoparallel plane (from 0 to $315^{\circ}$ in steps of $45^{\circ}$, where $0^{\circ}$ is rightward and $90^{\circ}$ is upward motion on the screen) and either five or nine binocular disparities (ranging from -3.2 to $3.2^{\circ}$ in steps of 1.6 or $0.8^{\circ}$, respectively). All directions and disparities were randomly interleaved in a block of trials, for a total of either 40 or 72 distinct stimulus combinations. Unlike the heading tuning protocol, stimuli here consisted of a planar sheet of dots, moving at constant speed, and each trial lasted $1 \mathrm{~s}$. Monkeys viewed the random-dot stimuli while maintaining fixation on a small yellow spot on the screen $\left(0.3^{\circ}\right)$. A small patch $\left(3^{\circ} \times 3^{\circ}\right.$, occluding the motion stimulus) of stationary dots was presented around the fixation point at zero disparity to help anchor vergence on the plane of the display screen.

For approximately half of the cells tested (MSTd, $n=41$; VIP, $n=73$ ), dots in the disparity-direction tuning protocol moved at the preferred speed of the neuron, which was determined by measuring a speed tuning curve using speeds of $0,0.5,1,2,4,8,16,32$, and $64^{\circ} / \mathrm{s}$. For these cells, the direction-disparity protocol consisted of nine disparities by eight directions by five repetitions plus five null trials to assess spontaneous activity (365 trials total). For the remaining MSTd neurons $(n=62)$, the disparity-direction tuning protocol (five disparities, eight directions) was delivered at two speeds: 8 and $64 \%$, randomly interleaved within a block of trials (for a total of $5 \times 8 \times 5 \times 2+5=405$ trials). Whenever cell isolation was maintained $(n=35)$, a separate block of trials also tested disparity-direction tuning at a speed of $2 \%$. In general, dot diameter was $0.4^{\circ}$ and dot density was 0.002 dots $\cdot$ degree $^{-2}$, which corresponds to $\sim 16$ dots on the screen at one time. When cell isolation could be maintained $(n=34)$, the disparity-direction protocol was also repeated at a higher dot density of 0.01 dots $\cdot$ degree $^{-2}(\sim 80$ dots on our display at a time), which was close to that used by Roy and Wurtz (1992). Because VIP cells generally preferred the highest speed tested (Colby et al., 1993), 28 VIP neurons were tested with the disparity-direction tuning protocol at the highest speed $\left(64^{\circ} / \mathrm{s}\right)$ only. Data were included in our analyses only if at least three repetitions of each stimulus were completed. At least five repetitions were completed for 135 of the 204 neurons that were included in the analysis, with the remaining 69 neurons having three or four repetitions.

\section{Data analysis}

Heading tuning protocol. Quantitative data analyses were performed offline using custom-written scripts in Matlab (MathWorks). For the heading protocol, mean firing rates were computed during the middle $1 \mathrm{~s}$ interval of each stimulus presentation, and an ANOVA $(p<0.05)$ was used to assess the statistical significance of directional selectivity for each stimulus condition. For neurons with significant heading tuning for both the visual and vestibular conditions, mean responses were plotted as a function of azimuth and elevation to create $3 \mathrm{D}$ tuning functions (for cells tested with the full set of heading vectors). To plot these spherical data on Cartesian axes, the data were transformed using the Lambert cylindrical equal-area projection (Fig. $2 A, B$ ) (for details, see Gu et al., 2006). In this flattened representation, the abscissa represents the azimuth angle, and the ordinate corresponds to a sinusoidally transformed version of the elevation angle.

For neurons tested with all 26 stimulus directions, the preferred 3D heading for each stimulus condition was described by the azimuth and elevation of the vector sum of the individual responses. For neurons tested with the reduced heading tuning protocol, only the preferred direction in the horizontal plane was computed by the vector sum. For multisensory cells with significant heading tuning in both the vestibular and visual conditions, we then computed the absolute difference in preferred direction, such that neurons could be characterized as "congruent" ( $\mid \Delta$ preferred heading $\left.\mid<90^{\circ}\right)$ or "opposite" ( $\mid \Delta$ preferred heading $\mid>90^{\circ}$ ).

Disparity-direction tuning. To compare our findings with the results of Roy et al. (1992), we computed the firing rate between 400 and $1000 \mathrm{~ms}$ after stimulus onset for each trial. We excluded the response in the first $400 \mathrm{~ms}$ to avoid transient responses to stimulus onset and to be consistent with Roy et al. (1992); note, however, that all of our main findings were unchanged if we analyzed neural responses over the full duration of the stimulus period. A two-way ANOVA was then used to test for a significant main effect of binocular disparity, a main effect of direction, and/or a significant interaction.

Tuning strength and Gabor fits. To measure disparity tuning strength, we computed a disparity discrimination index (DDI) for each neuron and each direction of motion as follows:

$$
\mathrm{DDI}=\frac{R_{\max }-R_{\min }}{R_{\max }-R_{\min }+2 \sqrt{\mathrm{SSE}} /(N-M)^{\prime}}
$$

where $R_{\max }$ and $R_{\min }$ are the mean responses to the most effective and least effective disparities, respectively, SSE is the sum squared error around the mean responses, $N$ is the number of observations (trials), and $M$ is the number of disparity values tested. Note that the DDI characterizes the ability of a neuron to discriminate changes in the disparity stimulus relative to its intrinsic level of response variability (Prince et al., 2002; DeAngelis and Uka, 2003).

For each neuron, we chose the direction of motion with maximal DDI and performed an ANOVA on the disparity tuning curve for that direction. For cells with significant disparity tuning (ANOVA, $p<0.01$ ), disparity tuning curves were fit with a Gabor function, as done previously 
for MT (DeAngelis and Uka, 2003; Palanca and DeAngelis, 2003; Chowdhury et al., 2008), V3/ V3A (Anzai et al., 2011), and V1 cells (Ohzawa et al., 1997; Prince et al., 2002):

$$
\begin{aligned}
R(d)= & R_{0}+A \times e^{-0.5\left(\left(d-d_{0}\right)^{2} / \sigma^{2}\right)} \\
& \times \cos \left(2 \pi f\left(d-d_{0}\right)+\Phi\right),
\end{aligned}
$$

where $R_{o}$ is the baseline response, $A$ is the response amplitude, $d$ is the stimulus disparity, $d_{o}$ is the disparity at the peak of the Gaussian envelope, and $\sigma$ is the Gaussian width. The phase of the Gabor function, relative to the center of the Gaussian envelope, is controlled by the parameter $\Phi$. Thus, the fit had five free parameters $\left(R_{o}, A, d_{o}, \sigma\right.$, and $\left.\Phi\right)$, whereas the disparity frequency, $f$, was determined from a Fourier transform of the raw tuning curve (DeAngelis and Uka, 2003). In addition, the Gaussian center location, $d_{o}$, was constrained to lie within the range of disparities tested. For each cell, the preferred disparity was computed from the peak of the Gabor fit. Only cells with good fits $\left(R^{2}>0.8\right)$ have been included in the analysis of Figure 10.

Most MSTd and VIP neurons had monotonic tuning for disparity over the range tested $\left(-3.2\right.$ to $\left.+3.2^{\circ}\right)$, with maximal responses often occurring at one of the largest near or far disparities presented. If an even larger range of disparities were tested, it is likely that tuning peaks would have been observed for some neurons. As a result, our Fourier analysis method may have overestimated the disparity frequency parameter, $f$, for some neurons. Note, however, that disparity frequencies in MSTd and VIP were substantially lower than those in area MT, such that any overestimation of $f$ would not undermine our conclusions regarding the differences in scale of disparity tuning between MSTd/VIP and MT (see Fig. 10).

Depth-sign selectivity. Because most MSTd/ VIP cells preferred large far or near disparities (few cells were tuned to disparities near $0^{\circ}$ ), we also quantified the overall selectivity for depth sign, using the depth sign discrimination index (DSDI) (Nadler et al., 2008, 2009):

$$
\mathrm{DSDI}=\frac{1}{n} \sum_{i=1}^{n} \frac{R_{\mathrm{far}(i)}-R_{\mathrm{near}(i)}}{\left|R_{\mathrm{far}(i)}-R_{\text {near }(i)}\right|+\sigma_{\mathrm{avg}}} .
$$

For each pair of depths symmetric around 0 (e.g., $\pm 1.6^{\circ}$ ), we calculated the difference in response between far $\left(R_{\mathrm{far}}\right)$ and near $\left(R_{\text {near }}\right)$ disparities, relative to response variability $\left(\sigma_{\mathrm{avg}}\right.$, the average standard deviation of the two responses). Then, we averaged the two or four pairs of depths tested to obtain the DSDI value for each neuron. The DSDI metric quantifies the cell's overall preference for near versus far disparities relative to the noise level of the responses; it ranges from -1 (strong near preference) to +1 (strong far preference) and was computed separately for each stimulus direction. To determine whether each DSDI was significantly different from 0 , we performed a permutation test by randomly shuffling the sign of each disparity (1000 permutations, $p<0.01$ ). We also computed a "global" DSDI across all stimulus directions after $z$-scoring the responses for each direction and combining across directions.

Two types of interactions between disparity and direction selectivity were classified: (1) DDD cells were defined when (i) either the main effect of disparity or the disparity by direction interaction was significant in the two-way ANOVA $(p<0.01)$, and (ii) there were at least two directions of
A Vestibular B Visual
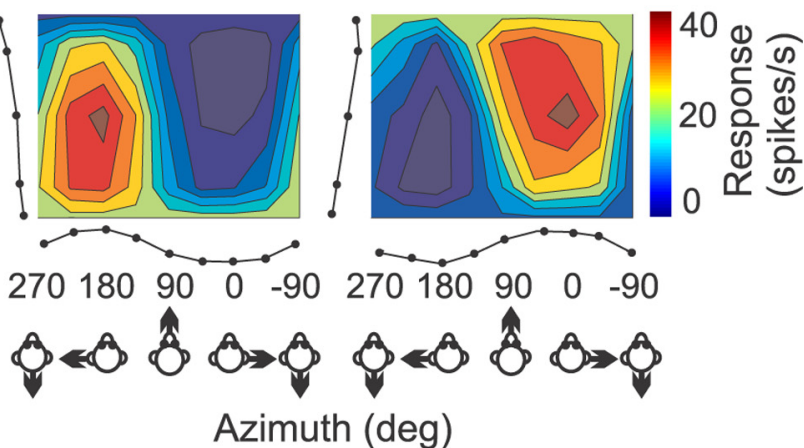

C

$\rightarrow-3.2^{\circ}$

- $-1.6^{\circ}$

$\rightarrow 0^{\circ}$

$\rightarrow 1.6^{\circ}$ $\rightarrow 3.2^{\circ}$

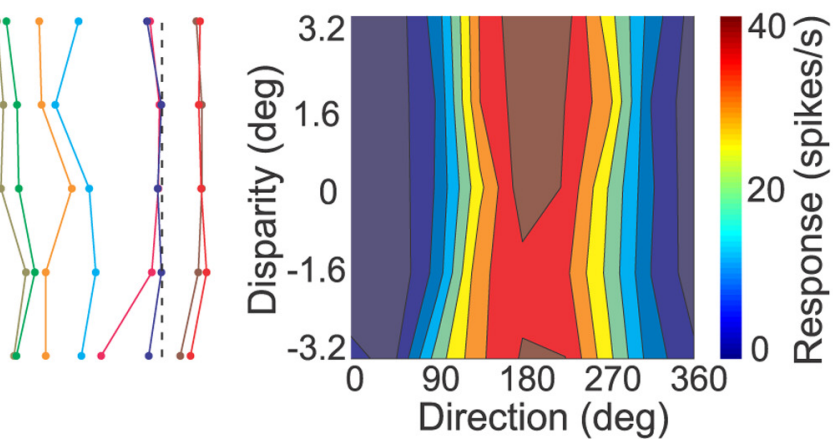

$\begin{array}{lll}90 & 180 & 270 \\ \text { Direction } & (\mathrm{deg})\end{array}$

Figure 2. 3D heading tuning and joint disparity-direction tuning for an "opposite" MSTd neuron. $A, B, 3 D$ heading tuning is shown for the vestibular $(\boldsymbol{A})$ and visual $(\boldsymbol{B})$ stimulus conditions as color contour maps of mean firing rate as a function of azimuth

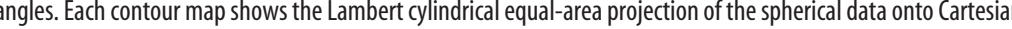
along the margins of each color map illustrate mean firing rates plotted as a function of either elevation or azimuth (averaged

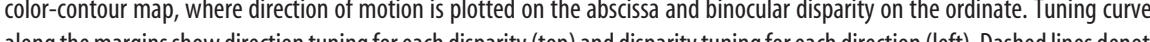
the margins show direction tuning for each disparity (top) and disparity tuning for each direction (left). Dashed lines denote

motion for which DSDI values were significantly different from zero $(p<0.01)$ and opposite in sign. These criteria identified neurons for which the depth-sign preference (near or far) reversed across directions of motion. (2) Non-DDD cells had a significant main effect of disparity in the two-way ANOVA $(p<0.01)$, but did not show a significant reversal in the sign of the DSDI across directions.

To summarize how the DSDI varied as a function of motion direction across the population of neurons, we plotted the DSDI as a function of direction of motion for each neuron and shifted this curve horizontally for each neuron (if needed) such that the direction having a maximal positive DSDI was aligned for each cell. This was done by computing the cross-correlation between each cell's DSDI curve and a sinusoidal function, $f(x)=\sin (x)$. The shift leading to a maximal correlation coefficient was used to align the curves across neurons. For non-DDD cells, the global DSDI metric was used to describe disparity selectivity across the population. Error bars in all plots illustrate SEM, unless noted otherwise.

\section{Results}

We recorded from 103 MSTd neurons (from four monkeys) and 101 VIP neurons (from three monkeys), all of which were significantly tuned for heading defined by optic flow (see Materials and Methods). Each neuron was first characterized as unisensory 


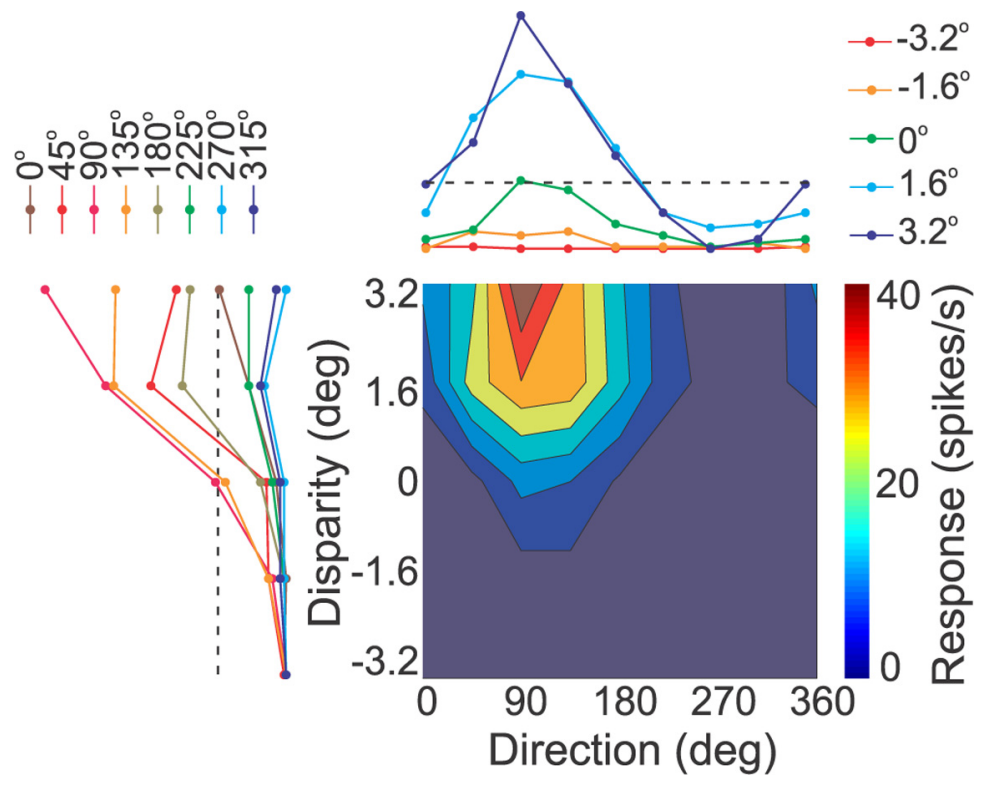

Figure 3. Joint direction-disparity tuning profile for a non-DDD, disparity-tuned, congruent MSTd cell. For this neuron, direction and disparity tuning are essentially separable, such that disparity tuning is similar for different directions and direction tuning is similar across disparities. Global DSDI $=0.707$ ( $p<0.001$, permutation test). The format is as in Figure $2 C$.

sented at five or nine different binocular disparities, for a total of 45 or 72 disparitydirection combinations (see Materials and Methods). Figure $2 C$ shows the joint disparity-direction tuning of the example cell, illustrated as a color-contour map, where the abscissa specifies motion direction $\left(0-360^{\circ}\right)$ and the ordinate represents horizontal binocular disparity $(0, \pm 1.6$, and $\left.\pm 3.2^{\circ}\right)$. This example neuron shows strong direction tuning at each disparity tested, but was not significantly disparity selective (main effect of disparity, twoway ANOVA, $p=0.14$ ). Thus, this particular MSTd neuron did not respond differently to moving random dots at different horizontal disparities.

Three different patterns of interaction of disparity and direction are illustrated by the example neurons in Figures 3 and 4. Figure 3 shows data for a congruent cell from area MSTd, which was selective for both direction of motion and binocular disparity (two-way ANOVA, $p<0.001$ for both main effects). The cell preferred

(visual only) or multisensory (significantly tuned to both visual and vestibular heading stimuli) based on responses measured during a heading tuning protocol (Gu et al., 2006) (see Materials and Methods). This protocol consisted of either real (vestibular condition) or visually simulated (visual condition) straight translational movements along either 26 possible directions sampled evenly on a sphere (Gu et al., 2006) or eight directions sampled within the horizontal plane. Inertial motion of the subject was achieved via a motion platform, and optic flow was presented by a projector mounted on the motion platform.

Multisensory neurons (MSTd, 42/103; VIP, 37/101) were significantly tuned (ANOVA, $p<0.05$ ) to heading for both visual and vestibular stimuli, as illustrated by the example cell in Figure 2, $A$ and $B$. The $3 \mathrm{D}$ heading tuning profile for each stimulus condition is shown as a color-contour map in which mean firing rate is plotted as a function of azimuth (abscissa) and elevation (ordinate). This cell exhibited broad, roughly sinusoidal tuning during inertial motion, with a heading preference at $192^{\circ}$ azimuth and $25^{\circ}$ elevation, corresponding to a leftward and slightly downward trajectory (Fig. $2 \mathrm{~A}$, vestibular condition). The heading preference in response to optic flow was approximately opposite, at $16^{\circ}$ azimuth and $-28^{\circ}$ elevation, corresponding to a rightward and slightly upward trajectory (Fig. $2 B$, visual condition). Thus, this is an example of an "opposite" cell (Gu et al., 2006; Takahashi et al., 2007; Chen et al., 2011b).

Across the population of neurons, heading tuning was classified as congruent or opposite by computing the absolute difference in heading preference $(\mid \Delta$ preferred heading $\mid)$ between responses to the two modalities, an angle that varies between 0 and $180^{\circ}$. In this context, congruent cells were defined as having $\mid \Delta$ preferred heading $\mid<90^{\circ}$, and opposite cells were defined as having $\mid \Delta$ preferred heading $\mid \geq 90^{\circ}$.

\section{Examples of joint disparity-direction tuning}

Once a cell was characterized as visual only or multisensory (either congruent or opposite), we measured the joint disparity and direction tuning of the neuron using a protocol in which eight directions of motion, spaced $45^{\circ}$ apart in the frontoparallel plane, were pre- the same binocular disparities for all motion directions and had the same preferred direction for all disparities (Fig. 3, left and top curves, respectively). In other words, direction and disparity tuning were separable for this neuron. For disparity-selective cells, we quantified selectivity for near versus far depth using a DSDI (Eq. 3), which varies from -1 (strong near preference) to +1 (strong far preference). For the neuron in Figure 3, DSDI values measured for each motion direction ranged from 0.597 to 0.884 , with a global DSDI of 0.707 . Responses like those seen in Figure 3 were common among our sample of disparity-selective neurons.

A different type of direction-disparity interaction is shown by the example neuron of Figure $4 A$. The cell's overall disparity selectivity was not significant when pooled across directions (main effect of disparity, two-way ANOVA, $p=0.91$ ), but the disparity by direction interaction was highly significant $(p<$ 0.001). Moreover, this cell showed a clear reversal in disparity preference for opposite motion directions. Specifically, the cell preferred near disparities (DSDI $<0$ ) for directions 270, 0, and $315^{\circ}$ (Fig. $4 A$, left), but preferred far disparities (DSDI $>0$ ) for directions 90,135 , and $180^{\circ}$. Analogously, this cell's direction preference also depended sharply on binocular disparity: for far disparities, the cell preferred motion that was upward and to the left on the screen (Fig. 4A, top), whereas for near disparities the cell preferred downward/rightward motion. We refer to this property as direction-dependent disparity tuning (for classification criteria, see Materials and Methods; for notes on interpretation, see Discussion). This sort of interaction was reported previously in MSTd (Roy and Wurtz, 1990; Roy et al., 1992) (see Discussion).

The cell illustrated in Figure $4 B$ is another DDD cell, but one with a qualitatively different pattern of results. For this neuron, the depth-sign preference (near/far) again reversed as a function of motion direction, such that the cell preferred far disparities for directions 0,45 , and $315^{\circ}$ (Fig. $4 B$, left), but near disparities for most other directions. However, unlike the cell in Figure $4 A$, the preferred direction of motion was consistent across all binocular disparities (Fig. $4 B$, top). This was the dominant type of DDD 
tuning encountered in our sample of neurons, and this pattern of results occurs because the disparity tuning curves for different directions of motion do not cross (Fig. $4 B$, left), even as the disparity preference reverses. Note that the two DDD cells in Figure 4 are both unisensory (visualonly) MSTd neurons. Figure $4 C$ summarizes how the DSDI changes with motion direction for the three disparity-selective exemplar neurons in Figures 3 and 4, A and $B$. For the non-DDD cell of Figure 3, the DSDI is fairly constant across directions. In contrast, the DSDI varies in a sinusoidal-like manner for the two DDD cells of Figure 4.

\section{Population properties}

Across our sample of MSTd neurons, 72 of $103(70 \%)$ were disparity selective, and these were classified as non-DDD $(n=49$, $48 \%)$ or DDD $(23,22 \%)$ cells (Table 1$)$. Most DDD cells in MSTd (17/23) were unisensory, visual-only neurons, which did not respond to vestibular stimulation. Indeed, DDD cells constituted 37\% (17 of 46) of all disparity-selective, visual-only cells. In contrast, only six DDD cells were multisensory, and these were evenly split between congruent and opposite cells. Only 5 of 23 DDD neurons, all of which were unisensory (visual-only) cells, reversed their disparity preference across motion directions and reversed their direction preference across disparities (Fig. 4A). Thus, the large majority (18 of $23,78 \%$ ) of DDD cells maintained their direction preference across disparities, while the disparity preference changed with direction (Fig. $4 B$ ).

Results from VIP were markedly different from MSTd (Table 1). First, there were fewer (41 of 101, 41\%) disparityselective cells in VIP. Second, only five of these neurons fulfilled the criteria to be DDD cells, and none of these cells clearly reversed their direction preference across disparities. The vast majority (36 of 41 , $88 \%)$ of disparity-selective VIP neurons were non-DDD cells. This difference in the incidence of DDD cells between MSTd and VIP was statistically significant $(p<$ 0.001, $\chi^{2}$ test).

Figure 5 summarizes how the DSDI varied as a function of motion direction for DDD and non-DDD cells in MSTd and VIP. To combine data across neurons, the DSDI versus direction curve for each cell was shifted along the abscissa to align the data such that the peak DSDI for all cells occurred at a direction of $90^{\circ}$ (see Materials and Methods). As illustrated in Figure 5, $A$ and $B$, the average DSDI of DDD cells depended strongly on motion
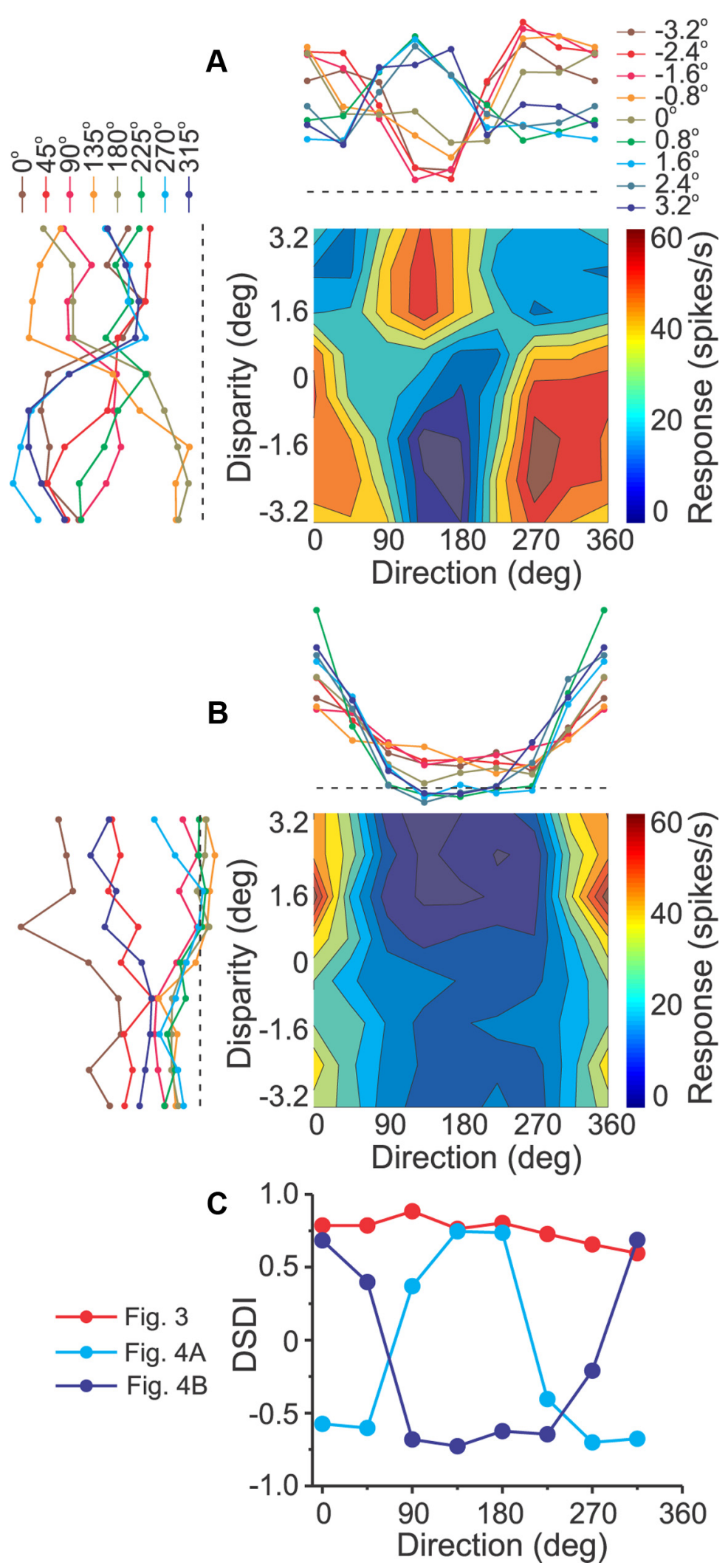

Figure 4. Examples of two DDD neurons from area MSTd. $\boldsymbol{A}$, For this cell, disparity preference reversed for opposite motion directions (left), and direction preference reversed for near versus far disparities (top). $\boldsymbol{B}$, For this neuron, disparity preference reversed for opposite motion directions (left), but direction preference did not reverse for near versus far disparities (top). The format is as in Figures 2 and 3. $\boldsymbol{C}$, The DSDI of the three disparity-selective example neurons (from Fig. 3 and $\boldsymbol{A}, \boldsymbol{B}$ ) is plotted as a function of motion direction. For non-DDD cells like the one from Figure 3, the DSDI changes little with direction of motion (red). For DDD cells (cyan, blue), the DSDI shows a strong reversal in sign across different motion directions. 
Table 1. Statistics of disparity tuning in MSTd and VIP

\begin{tabular}{llll}
\hline & & \multicolumn{2}{l}{ Non-DDD neurons } \\
\cline { 4 - 4 } & DDD neurons & $p_{\text {disparity }}<0.01$ & $p_{\text {dispaity }} \geq 0.01$ \\
\hline MSTd $(n=103)$ & & \\
Unisensory neurons $(n=61)$ & 17 & 29 & 15 \\
$\quad$ Multisensory neurons & & & \\
$\quad$ Congruent cells $(n=17)$ & 3 & 9 & 5 \\
$\quad$ Opposite cells $(n=25)$ & 3 & 11 & 11 \\
Total & $23,22 \%$ & $49,48 \%$ & $31,30 \%$ \\
VIP $(n=101)$ & & & \\
Unisensory neurons $(n=64)$ & 4 & 26 & 34 \\
Multisensory neurons & & & \\
$\quad$ Congruent cells $(n=12)$ & 1 & 5 & 9 \\
$\quad$ Opposite cells $(n=20)$ & 0 & 5 & 17 \\
Total & $5,5 \%$ & $36,36 \%$ & $60,59 \%$ \\
\hline
\end{tabular}
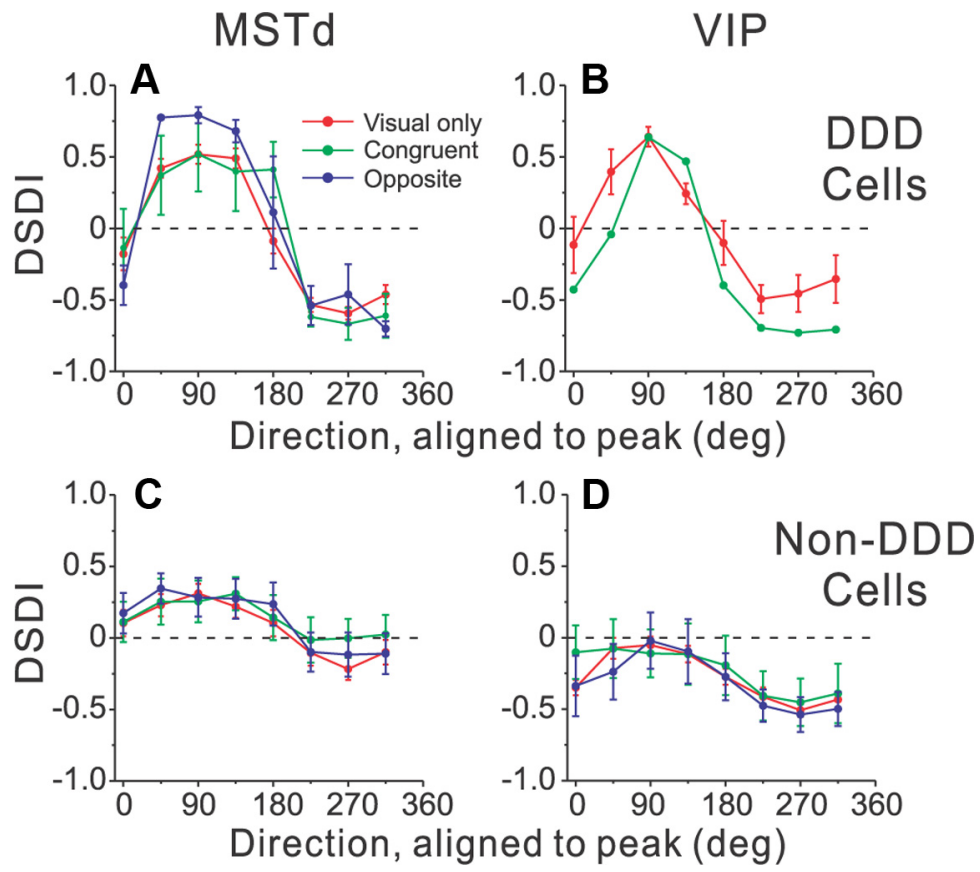

Figure 5. Population summary of dependence of the DSDI on motion direction. $A-D$, Data are averaged across all disparityselective neurons from MSTd $(\boldsymbol{A}, \boldsymbol{C})$ and VIP $(\boldsymbol{B}, \boldsymbol{D})$, and are shown separately for $\operatorname{DDD}(\boldsymbol{A}, \boldsymbol{B})$ and non-DDD $(\boldsymbol{C}, \boldsymbol{D})$ cells. Data are color coded to represent visual-only neurons (red) and multisensory congruent (green) or opposite (blue) cells. Before averages were computed, data for each neuron were horizontally shifted and wrapped such that the peaks of all DSDI curves aligned at a direction of $90^{\circ}$. The necessary shift for each neuron was determined by computing a cross-correlation between the DSDI versus direction curve and a sinusoid (see Materials and Methods). The numbers of neurons contributing to each summary curve are as follows: $A$, 3 congruent cells, 3 opposite cells, 17 visual-only cells; $\boldsymbol{B}, 1$ congruent cell, 4 visual-only cells; $\boldsymbol{C}, 9$ congruent cells, 11 opposite cells, 29 visual-only cells; $\boldsymbol{D}, 5$ congruent cells, 5 opposite cells, 26 visual-only cells.

direction, whereas non-DDD cells showed a much more modest dependence (Fig. 5C,D). To quantify this distinction, we calculated the difference in DSDI between two opposite directions, 90 and $270^{\circ}$. This difference averaged $1.14 \pm 0.07$ for DDD cells in MSTd, versus $0.43 \pm 0.05$ for non-DDD cells $(p<0.001$, Wilcoxon test). Similarly, for VIP, the average difference in DSDI was $1.15 \pm 0.08$ for DDD cells versus $0.45 \pm 0.04$ for non-DDD cells $(p<0.001)$.

Note that some of the direction dependence of the DSDI may result simply from our procedure of aligning all curves at their peak DSDI. To assess this, we permuted the data across directions for each neuron and computed the distribution of DSDI values expected by chance when any true directional dependence of the DSDI is destroyed by permutation. For non-DDD cells from both MSTd and VIP, the average difference in DSDI between 90 and $270^{\circ}$ was $\sim 0.3$ following permutation. Thus, although the modulation of the DSDI with direction for non-DDD cells was significantly greater than that expected by chance $(p<0.01)$, this modulation was quite modest relative to that exhibited by DDD neurons.

As noted above, most DDD cells did not reverse both their direction and disparity preferences. We performed an additional analysis to quantify the incidence of these effects across the population of DDD neurons. For each DDD cell, we found the pair of opposite directions $\left(180^{\circ}\right.$ apart $)$ that produced the maximal absolute difference in DSDI. For this purpose, we refer to the direction with the larger maximal response as "preferred" and the opposite direction as "null" (Fig. 6A). For near disparities (less than or equal to $-1.6^{\circ}$ ), we computed the average difference in firing rate between the preferred and null direction curves, and we plotted this value on the abscissa of the scatter plot in Figure 6. Similarly, for far disparities $\left(\geq+1.6^{\circ}\right)$, we plotted the average response difference between preferred and null directions on the ordinate of the scatter plot. Thus, data points that fall in the upper-left or lowerright quadrants of the scatter plot indicate neurons that reversed both direction preference and disparity preference, such as the examples shown in Figure 6, $A$ and $E$. Data points that fall in the upper-right or lower-left quadrants indicate neurons that reverse disparity preference but not direction preference (Fig. 6B,C). Clearly most neurons fall in the upper-right quadrant indicating that they do not reverse direction preference.

We now return to non-DDD neurons, to summarize their disparity and velocity tuning properties. For disparity-selective non-DDD cells, we computed a global DSDI value across all motion directions to summarize disparity selectivity (see Materials and Methods). The mean DSDI for non-DDD cells in VIP $(-0.317 \pm 0.050$, $\mathrm{SE}$ ) was significantly less (Wilcoxon rank test, $p<0.001)$ than that for MSTd $(0.100 \pm 0.062)$, as illustrated in Figure 7 , $A$ and $B$. In MSTd, 22 cells had DSDI values significantly larger than zero, and 16 cells had DSDI values significantly less than 0 . In contrast, only 4 cells in VIP had a significantly positive DSDI, whereas 29 cells had significantly negative DSDI values. This difference indicates a significant shift toward near disparity preferences in VIP (Colby et al., 1993). There was no significant difference between DSDI distributions of multisensory and visual-only neurons in either MSTd $\left(p=0.83, \chi^{2}\right.$ test) or VIP ( $p=0.28, \chi^{2}$ test). Importantly, both near- and farpreferring cells were encountered in roughly equal proportions among multisensory cells, with no significant difference in the distribution of the DSDI between congruent and opposite cells (MSTd, $p=0.84, \chi^{2}$ test; VIP, $p=0.86$ ). It was clearly not the case that congruent cells generally had near depth preferences and opposite cells typically preferred far depths, as would be expected from the motion parallax hypothesis (see Introduction).

In addition to the DSDI, speed tuning also differed between VIP and MSTd ( $p<0.001, \chi^{2}$ test), as illustrated in Figure 7, $C$ 
and $D$. Specifically, VIP cells preferred higher speeds than MSTd cells. Among 41 MSTd neurons tested, 14 (34\%) preferred the highest speed $(64 \%)$, and $10(24 \%)$ preferred the second highest speed $(32 \%$ s). In contrast, 64 of 73 (88\%) of VIP cells preferred $64 \%$, and $7(10 \%)$ preferred $32 \%$ s. There was no significant difference in speed preference between multisensory and unisensory neurons for either MSTd $\left(p=0.19, \chi^{2}\right.$ test $)$ or VIP $\left(p=0.26, \chi^{2}\right.$ test).

We also pooled all non-DDD data from MSTd and VIP together to test whether there is a significant correlation between the DSDI and preferred speed. There was indeed such a correlation between the global DSDI of non-DDD cells and speed preference $(r=-0.46 ; p=0.005$; Spearman rank correlation, $n=36$ ). Neurons that preferred slow speeds also preferred far depths, and vice versa. This correlation was driven partially by differences between areas given that VIP cells prefer high speeds and have near disparity preferences. Nevertheless, there was still a marginally significant relationship between the DSDI and speed preference when only MSTd cells were considered $(r=$ $-0.49 ; p=0.063$; Spearman rank correlation, $n=15$ ).

Other stimulus parameters, like dot density ( $p=0.38$, Wilcoxon matchedpairs test $)$ and speed $\left(p=0.90, F_{(1,99)}=\right.$ 0.033 , ANCOVA), had no effect on the depth-sign selectivity of MSTd neurons, as illustrated in Figure 8, $A$ and $B$, respectively (shown for non-DDD cells only).

\section{Comparison of disparity tuning properties among areas MSTd, VIP, and MT}

To quantify disparity tuning curves for MSTd and VIP neurons, we computed a DDI (Eq. 1) for each of the eight directions of motion. The disparity tuning curve for the direction with the largest DDI was then fit with a Gabor function, as long as tuning was significant (ANOVA, $p<0.01$ ). These criteria were met for 63 MSTd and 39 VIP neurons. Of these, the disparity tuning curves of 55 MSTd cells and 29 VIP cells were well fit by a Gabor function $\left(R^{2}>0.8\right)$, and these responses were used for quantitative comparisons with area MT.

Figure 9 shows disparity tuning curves and Gabor fits for four example neurons, two DDD cells and one non-DDD cell from MSTd, as well as one non-DDD neuron from VIP. Data on the left show disparity tuning for the maximum DDI direction, with solid curves illustrating Gabor fits. Data on the right show tuning curves for the opposite direction of motion, along with Gabor fits when tuning was significant (ANOVA, $p<0.01$ ). The majority of MSTd and VIP cells preferred either near or far disparities and had monotonic tuning curves within the range of disparities tested. The DDD cell of Figure 9A preferred near disparities for the max DDI direction and far disparities for the opposite direction, with monotonic tuning for both directions. In contrast, the DDD cell of Figure $9 B$ shows opposite depth-sign preferences and has nonmonotonic tuning. The non-DDD MSTd cell of Figure $9 C$ shows clearly peaked tuning with a preference near zero dis- parity, which was not common in MSTd or VIP. Finally, the non-DDD VIP cell of Figure 9D shows monotonic tuning to near disparities for one direction of motion and no significant disparity tuning for the other direction.

Figure 10 summarizes the disparity tuning properties of MSTd (red) and VIP (blue) neurons and compares them with data from area MT (green) (DeAngelis and Uka, 2003). Note that all cells are included in Figure $10 A$, whereas only neurons with significant disparity tuning and good Gabor fits are included in Figures $10, B$ and $C$. Overall, the strength of disparity tuning in area MT (median DDI, 0.74$)$ is significantly greater $(p<0.001$, Mann-Whitney $U$ test) than that seen in MSTd and VIP (median DDI, 0.57 and 0.54 , respectively), whereas the difference between MSTd and VIP was marginal ( $p=0.028$, Mann-Whitney $U$ test). There was no significant correlation between DDI and speed preference for area MSTd ( $p=0.73$, Spearman rank correlation) or VIP $(p=0.46)$ separately, nor when data from these areas were pooled $(p=0.52)$. In contrast, DDI depended significantly on speed preference in MT, such that neurons preferring fast speeds tended to have weaker disparity selectivity (DeAngelis and Uka, 2003).

Striking differences between areas are evident in the range of disparity preferences and the breadth of tuning. As shown in Figure $10 B$, neurons in MSTd and VIP are tuned to a much broader range of disparities than cells in MT. VIP neurons show a strong bias toward near preferences (median, $-2.21^{\circ}$ ), whereas disparity preferences in MSTd are fairly balanced (median, $\left.0.02^{\circ}\right)$, and this difference is significant ( $p=0.003$, MannWhitney $U$ test). By comparison, disparity preferences in MT are much more tightly distributed around zero disparity, with a slight 

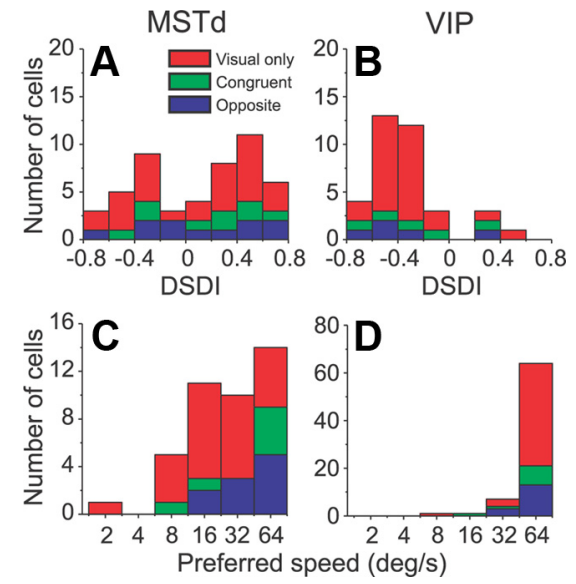

Figure 7. Population summary of the DSDI and speed preferences. $A, B$, Distributions of the global DSDI (computed across all motion directions) for disparity-selective non-DDD neurons from areas MSTd $(n=49)$ and VIP $(n=36)$. C, $D$, Distributions of preferred speed for MSTd $(n=41)$ and VIP $(n=73)$ neurons. Data are color coded to represent visual only neurons (red) and multisensory congruent (green) or opposite (blue) cells.
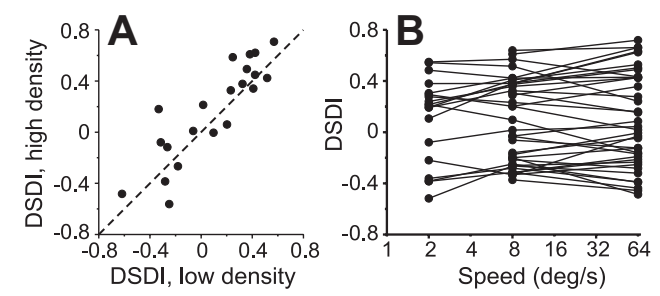

Figure 8. Dependence of the DSDI on dot density and stimulus speed. Data are shown for non-DDD cells from area MSTd. A, DSDI measured at a higher dot density $(0.01$ dots $\cdot$ degree $\left.{ }^{-2}\right)$ is plotted against the DSDI measured at the standard density $(0.002$ dots $\cdot$ degree $\left.^{-2} ; n=21\right) . B$, DSDl is measured as a function of stimulus speed for a subset of MSTd neurons $(n=38)$.

bias toward near preferences (median, $-0.16^{\circ}$ ) (DeAngelis and Uka, 2003). Figure $10 \mathrm{C}$ shows that median disparity frequencies in MSTd (0.11) and VIP ( 0.10$)$ were similar $(p=0.53)$, and were significantly lower than in MT $(0.29, p<0.001$, Mann-Whitney $U$ test $)$. The lower disparity frequencies in MSTd/VIP are consistent with the observation that disparity tuning in these areas was most commonly monotonic, with a preference for large far or near disparities.

It is important to be sure that these differences in disparity tuning parameters between areas are not an indirect effect of differences in receptive field eccentricity between recordings from the three areas. As shown in Figure $10 \mathrm{D}-\mathrm{F}$, eccentricities were indeed systematically smaller in the MT recordings than in MSTd/VIP. Note, however, that the greater range of disparity preferences and lower disparity frequencies observed in MSTd/ VIP cannot be attributed to eccentricity. Within the range of eccentricities sampled in all three areas $\left(\sim 10-25^{\circ}\right)$, disparity preferences in area MT are much more narrowly distributed than those in MSTd/VIP (Fig. 10E), and disparity frequencies in MT are substantially larger than those in MSTd/VIP (Fig. 10F).

\section{Discussion}

Motivated by the possibility that the congruency of visual/vestibular heading tuning might be correlated with stereoscopic depth preferences, we examined the binocular disparity tuning of neurons in areas MSTd and VIP in relation to heading selectivity. Many MSTd and VIP neurons showed monotonic disparity tuning with preferences for large near or far disparities, but we found
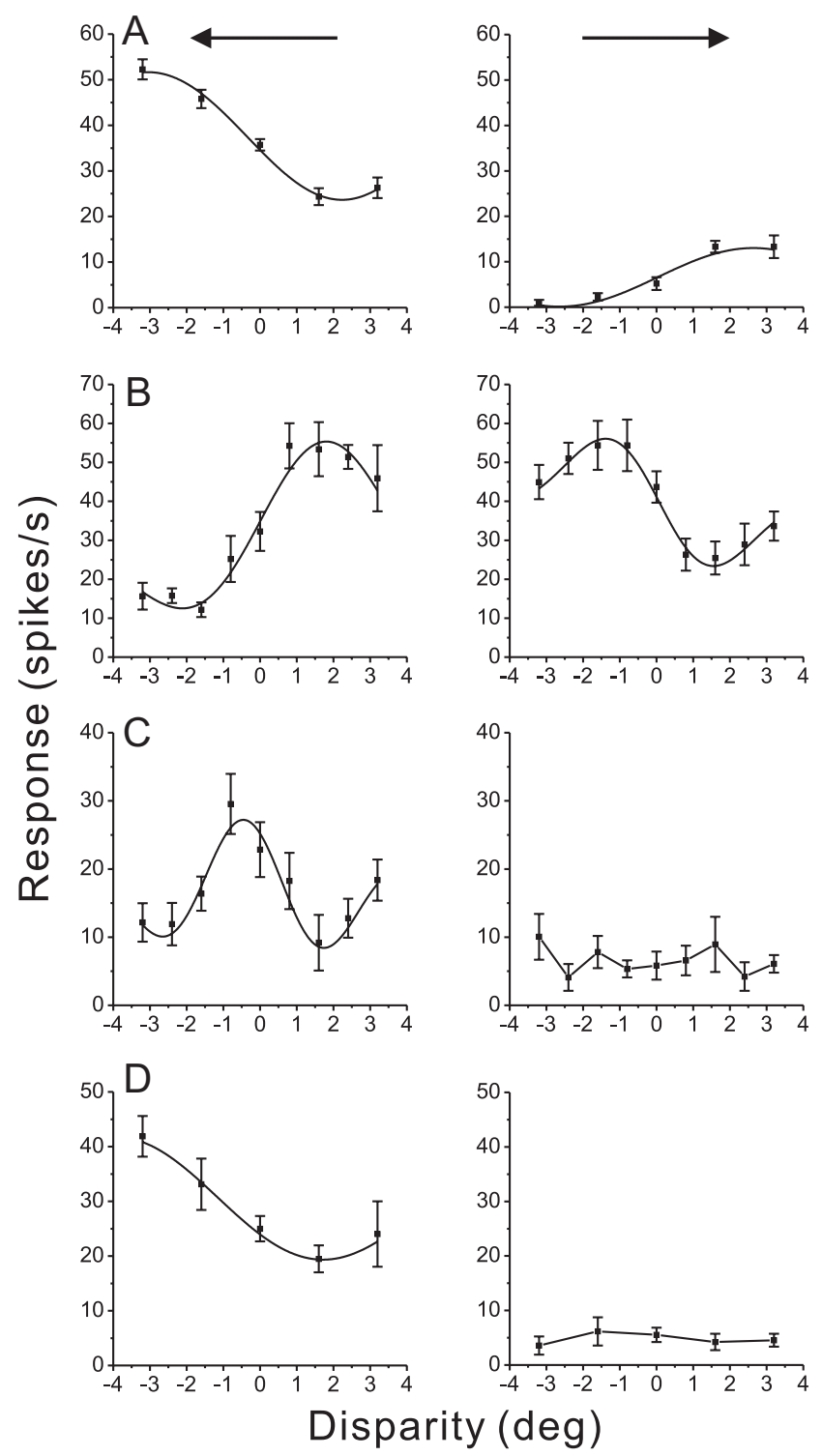

Figure 9. Example disparity tuning curves and Gabor fits for MSTd and VIP neurons. For each neuron/row, disparity tuning is shown for the direction of maximum DDI (left) and for the direction $180^{\circ}$ opposite to it (right). $\boldsymbol{A}-\boldsymbol{D}$, Data are shown for two DDD cells from MSTd $(\boldsymbol{A}, \boldsymbol{B})$, one non-DDD cell from MSTd $(\boldsymbol{C})$, and one non-DDD neuron from VIP $(\boldsymbol{D})$. Smooth curves represent Gabor function fits (see Materials and Methods). Gabor fits are shown for both directions of motion for DDD cells, but only for the maximum DDI direction for non-DDD cells.

no evidence that visual-vestibular congruency is related to depth preference. We also sought to understand how the DDD property may be related to tuning for self-motion. Although a substantial proportion of MSTd cells exhibit DDD properties (fewer in VIP), most DDD neurons do not appear to receive any vestibular input. This suggests that DDD cells may provide a distinct mechanism for detecting self-motion, one that is largely independent of vestibular input.

\section{Disparity tuning in MSTd and the DDD property}

Disparity selectivity was studied previously in both MSTd (Roy and Wurtz, 1990; Roy et al., 1992; Takemura et al., 2000, 2001) and MSTl (Eifuku and Wurtz, 1999). The overall incidence of disparity selectivity among MSTd cells reported here (70\%, based on ANOVA) is a bit lower than the $90 \%$ reported by Roy et al. (1992) based on nonstatistical criteria. Consistent with Roy et al. 

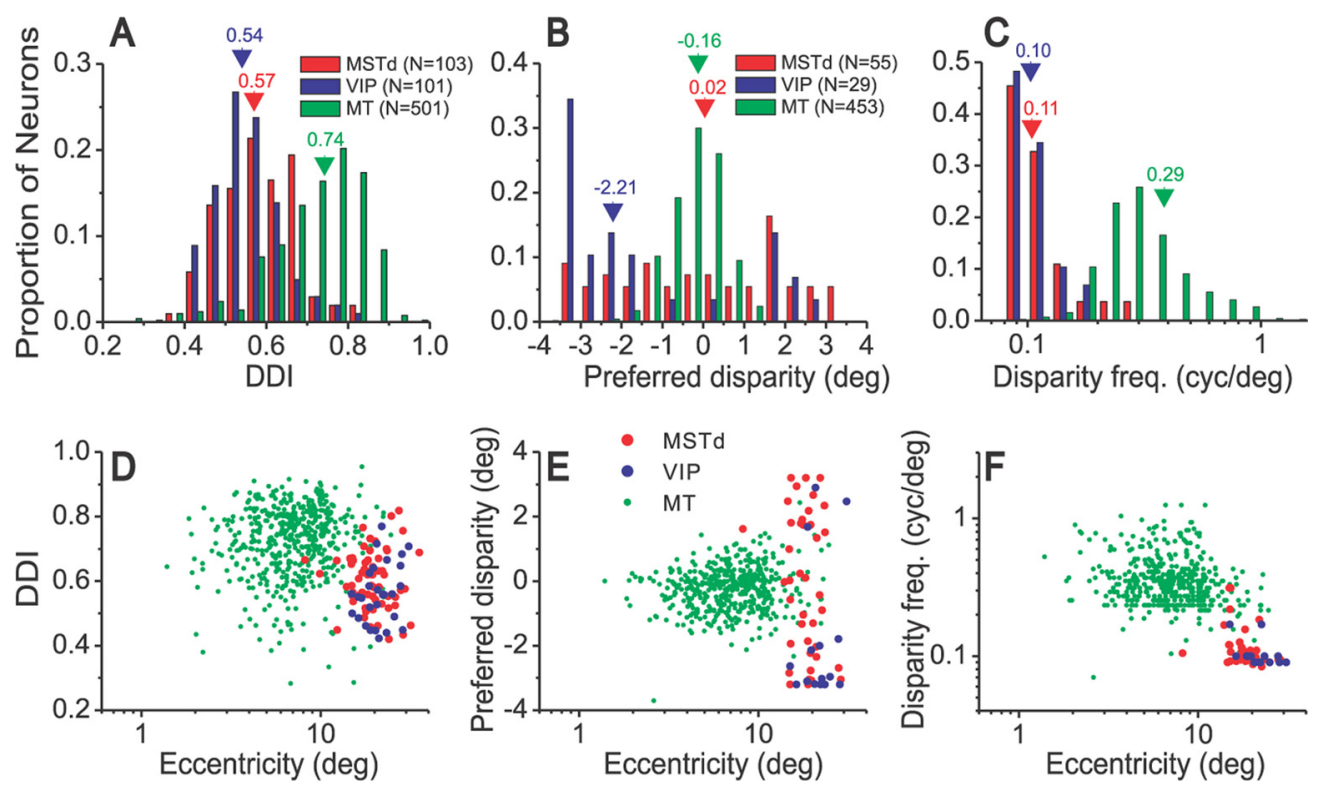

Figure 10. Comparison of disparity selectivity in areas MSTd, VIP, and MT. $\boldsymbol{A}-\boldsymbol{C}$, The top row shows distributions of the DDI $(\boldsymbol{A})$, preferred disparity $(\boldsymbol{B})$, and disparity frequency $(\boldsymbol{C})$ parameters. $\boldsymbol{A}$, Data for all neurons tested: 103 MSTd neurons (red), 101 VIP cells (blue), and 501 MT neurons (green). B, C, Data for 55 MSTd cells and 29 VIP neurons that had significant disparity tuning ( $p<0.01$, one-way ANOVA) for the max DDI direction and were well-fit by the Gabor function $\left(R^{2}>0.8\right)$ (see Materials and Methods). The MT data in $B$ and $C$ represent 453 MT neurons with significant disparity tuning $(p<0.01)$ (DeAngelis and Uka, 2003). Numbers above arrowheads show the median values for each distribution. $D$, DDI is plotted as a function of receptive field eccentricity for neurons from MT ( $n=501)$, MSTd $(n=65)$, and VIP $(n=28)$. $\boldsymbol{E}$, Preferred disparity as a function of eccentricity (MT, $n=453 ;$ MSTd, $n=41 ; V I P, n=15)$. $\boldsymbol{F}$, Disparity frequency as a function of eccentricity, for the same samples of neurons as in $\boldsymbol{E}$.

(1992), we find that most MSTd cells prefer either far (uncrossed) or near (crossed) disparities, in approximately equal proportions, with few neurons tuned to zero disparity.

Disparity tuning in MSTd is generally independent of motion speed (Fig. $8 \mathrm{~B}$ ), but often depends strongly on motion direction. Approximately one-third of disparity-tuned MSTd neurons in our sample reversed their disparity preference for opposite directions of motion (Fig. 4). However, the converse was seldom true; only 5 of 23 DDD neurons reversed their direction preference with disparity (Fig. 4A). Similar interactions between direction and disparity have been described previously by Roy and Wurtz (1990) and Roy et al. (1992), who emphasized the existence of neurons that changed their direction preference with disparity. They used the same acronym, DDD, to refer to "disparitydependent direction selectivity," even though disparity tuning was tested along just the preferred and antipreferred directions of motion. In contrast, we used a fully nested design in which all combinations of several disparities and directions were presented.

The incidence of significant direction-disparity interactions observed here (32\% of MSTd neurons) is similar to the $40 \%$ reported by Roy et al. (1992). However, it is not clear whether their criteria for identifying DDD cells were comparable to ours. Roy et al. (1992, p. 2490 ) state that "the $40 \%$ of MST neurons that are DDD cells ... respond to opposite directions of motion for opposite disparities." However, the example DDD neuron in their Figure $8 B$, like our Figure $4 B$, does not reverse its direction preference with disparity. Indeed, the vast majority of DDD cells in our sample (18 of 23) did not reverse their direction preference as disparity changed. Hence, we use the acronym DDD to refer to direction-dependent disparity tuning, rather than disparity-dependent direction tuning, as the former description applies to most cells with significant disparitydirection interactions, whereas the latter does not.

It is unclear whether our findings really differ from those of Roy and Wurtz (1990) and Roy et al. (1992), because they did not report how often preferred direction reversed with changes in disparity. However, some differences between their studies and ours are worth noting. First, Roy and Wurtz (1990) and Roy et al. (1992) recorded from both MSTd and MSTi (a region between MSTd and MSTl), whereas our recordings are likely to be only from MSTd. Second, we recorded from neurons with significant visual heading tuning, whereas Roy et al. (1992) recorded only from cells that were directionally selective in the frontoparallel plane. Thus, our sample might have included neurons selective for forward/backward translation that were not sampled by Roy and Wurtz (1990) and Roy et al. (1992). This is unlikely to be a major factor, however, because most MSTd cells prefer translation within the frontoparallel plane (Gu et al., 2006, 2010). Third, we used a full-field stimulus $\left(90 \times 90^{\circ}\right)$, whereas Roy and Wurtz (1990) and Roy et al. (1992) used smaller stimuli, typically $20 \times$ $20^{\circ}$. Finally, Roy and Wurtz (1990) and Roy et al. (1992) used relatively slow speeds of motion (up to $9 \%$ ), whereas we either used the cell's preferred speed or interleaved speeds of 8 and $64 \%$ s. However, this is not likely an important factor, as we found little dependence of disparity selectivity on speed or dot density (Fig. 8).

\section{Differences in disparity and speed tuning between MSTd, VIP, and MT}

Disparity tuning was least prominent in VIP, where $41 \%$ of cells were disparity selective and most preferred near disparities. Colby et al. (1993) reported that VIP neurons preferred near or ultranear stimuli, and that the near preference of some VIP cells persisted when one eye was occluded, suggesting involvement of monocular depth cues. In our stimuli, depth was defined exclusively by disparity, but the preponderance of near preferences remained. Notably, DDD cells were much less common in VIP than MSTd, consistent with the suggestion that VIP neurons mainly represent foreground motion (Colby et al., 1993).

We found robust differences in disparity tuning between MSTd, VIP and MT. First, $>90 \%$ of MT neurons were tuned for 
binocular disparity (DeAngelis and Uka, 2003), compared to $70 \%$ in MSTd and $41 \%$ in VIP. Correspondingly, average DDI values were substantially larger in MT than MSTd or VIP (Fig. $10 A)$. Second, disparity preferences in area MT were more centrally distributed around the plane of fixation, whereas most MSTd/VIP cells showed monotonic tuning with a preference for large far or near disparities. Thus, disparity selectivity changes markedly as signals flow downstream from area MT.

We found that speed tuning of most MSTd and VIP cells was monotonic over the range of speeds tested, with most cells preferring high speeds as seen in previous studies of MST (Kawano et al., 1994; Duffy and Wurtz, 1997; Churchland and Lisberger, 2005; Churchland et al., 2007). Speed tuning in VIP has been less well characterized, although Colby et al. (1993) reported that VIP neurons tend to prefer higher speeds than MT neurons. The emphasis on high speeds in MSTd and VIP contrasts with a roughly uniform distribution of speed preferences (on a logarithmic scale) in area MT (Nover et al., 2005).

\section{The motion parallax hypothesis and visual-vestibular congruency}

These experiments were motivated by the hypothesis that the existence of multisensory neurons with opposite visual and vestibular heading preferences might be explained by their depthsign preferences. Opposite cells were initially identified in experiments that used a head-fixed fixation target ( $\mathrm{Gu}$ et al., 2006; Takahashi et al., 2007), such that self-motion produces the same direction of retinal image motion for near and far objects (Fig. $1 B$ ). However, when fixation is maintained on a world-fixed target, self-translation produces opposite directions of retinal image motion for near and far objects (Fig. $1 A$, motion parallax). Thus, opposite cells could be driven maximally by self-motion if they prefer far depths, whereas congruent cells should prefer near depths in this hypothesis. In other words, both cell types might be "functionally congruent" when fixation is maintained on a world-fixed target. However, we did not find any consistent relationship between visual-vestibular congruency and depth-sign preferences (Fig. $7 A, B$ ). Thus, the motion parallax hypothesis does not appear to account for the existence of opposite cells. As speculated previously (Gu et al., 2008; Morgan et al., 2008), opposite cells may instead serve important roles in dissociating object motion from self-motion, a topic of current investigation in our laboratories.

\section{Function of DDD tuning and MSTd/VIP}

Roy et al. (1992) proposed that DDD cells contribute to signaling the direction of self-motion. Specifically, they postulated that the reversal of preferred direction for near versus far disparities reflects the ability of these neurons to respond to combinations of foreground and background motion consistent with self-translation during fixation of a world-fixed point. Our findings, however, call into question the notion of a privileged role of DDD cells in self-motion perception.

First, we found very few cells in MSTd $(\sim 5 \%)$ that reversed their direction preference with disparity, but many more that reversed their disparity preference as a function of direction. The latter, more abundant type do not respond best to opposite directions of image motion for near and far objects, and thus may not be as well suited to detecting observer movement as suggested previously (Roy et al., 1992). Second, DDD properties were much more commonly found in unisensory visual-only neurons than multisensory cells. If DDD cells are critical for self-motion perception, they might be expected to have congruent visual and vestibular heading tuning. However, this was not the case in either MSTd or VIP.

In summary, DDD neurons do not appear well suited to contribute to multisensory cue integration for self-motion perception. However, this does not exclude a role of DDD cells in processing self-motion, particularly the minority that reverse their direction preference with depth. One possibility is that these DDD cells may provide a parallel mechanism for estimating selfmotion when robust vestibular signals are not available to interact with optic flow. The functional contribution of DDD neurons to self-motion perception in trained animals thus clearly deserves further study.

\section{References}

Angelaki DE, Cullen KE (2008) Vestibular system: the many facets of a multimodal sense. Annu Rev Neurosci 31:125-150.

Anzai A, Chowdhury SA, DeAngelis GC (2011) Coding of stereoscopic depth information in visual areas V3 and V3A. J Neurosci 31:10270-10282.

Bremmer F, Kubischik M, Pekel M, Lappe M, Hoffmann KP (1999) Linear vestibular self-motion signals in monkey medial superior temporal area. Ann N Y Acad Sci 871:272-281.

Bremmer F, Klam F, Duhamel JR, Ben Hamed S, Graf W (2002) Visualvestibular interactive responses in the macaque ventral intraparietal area (VIP). Eur J Neurosci 16:1569-1586.

Chen A, DeAngelis GC, Angelaki DE (2011a) Convergence of vestibular and visual self-motion signals in an area of the posterior sylvian fissure. J Neurosci 31:11617-11627.

Chen A, DeAngelis GC, Angelaki DE (2011b) Representation of vestibular and visual cues to self-motion in ventral intraparietal (VIP) cortex. J Neurosci 31:12036-12052.

Chowdhury SA, Christiansen DL, Morgan ML, DeAngelis GC (2008) Effect of vertical disparities on depth representation in macaque monkeys: MT physiology and behavior. J Neurophysiol 99:876-887.

Churchland AK, Lisberger SG (2005) Relationship between extraretinal component of firing rate and eye speed in area MST of macaque monkeys. J Neurophysiol 94:2416-2426.

Churchland AK, Huang X, Lisberger SG (2007) Responses of neurons in the medial superior temporal visual area to apparent motion stimuli in macaque monkeys. J Neurophysiol 97:272-282.

Colby CL, Duhamel JR, Goldberg ME (1993) Ventral intraparietal area of the macaque: anatomic location and visual response properties. J Neurophysiol 69:902-914.

DeAngelis GC, Uka T (2003) Coding of horizontal disparity and velocity by MT neurons in the alert macaque. J Neurophysiol 89:1094-1111.

Duffy CJ (1998) MST neurons respond to optic flow and translational movement. J Neurophysiol 80:1816-1827.

Duffy CJ, Wurtz RH (1997) Medial superior temporal area neurons respond to speed patterns in optic flow. J Neurosci 17:2839-2851.

Eifuku S, Wurtz RH (1999) Response to motion in extrastriate area MSTl: disparity sensitivity. J Neurophysiol 82:2462-2475.

Gibson JJ (1950) The perception of the visual world. Cambridge, MA: Riverside.

Gu Y, Watkins PV, Angelaki DE, DeAngelis GC (2006) Visual and nonvisual contributions to three-dimensional heading selectivity in the medial superior temporal area. J Neurosci 26:73-85.

Gu Y, Angelaki DE, DeAngelis GC (2008) Neural correlates of multisensory cue integration in macaque MSTd. Nat Neurosci 11:1201-1210.

Gu Y, Fetsch CR, Adeyemo B, DeAngelis GC, Angelaki DE (2010) Decoding of MSTd population activity accounts for variations in the precision of heading perception. Neuron 66:596-609.

Judge SJ, Richmond BJ, Chu FC (1980) Implantation of magnetic search coils for measurement of eye position: an improved method. Vision Res 20:535-538.

Kawano K, Shidara M, Watanabe Y, Yamane S (1994) Neural activity in cortical area MST of alert monkey during ocular following responses. J Neurophysiol 71:2305-2324.

Morgan ML, DeAngelis GC, Angelaki DE (2008) Multisensory integration in macaque visual cortex depends on cue reliability. Neuron 59:662-673.

Nadler JW, Angelaki DE, DeAngelis GC (2008) A neural representation of depth from motion parallax in macaque visual cortex. Nature 452:642-645. 
Nadler JW, Nawrot M, Angelaki DE, DeAngelis GC (2009) MT neurons combine visual motion with a smooth eye movement signal to code depth-sign from motion parallax. Neuron 63:523-532.

Nover H, Anderson CH, DeAngelis GC (2005) A logarithmic, scale-invariant representation of speed in macaque middle temporal area accounts for speed discrimination performance. J Neurosci 25:10049-10060.

Ohzawa I, DeAngelis GC, Freeman RD (1997) Encoding of binocular disparity by complex cells in the cat's visual cortex. J Neurophysiol 77:2879-2909.

Page WK, Duffy CJ (2003) Heading representation in MST: sensory interactions and population encoding. J Neurophysiol 89:1994-2013.

Palanca BJ, DeAngelis GC (2003) Macaque middle temporal neurons signal depth in the absence of motion. J Neurosci 23:7647-7658.

Prince SJ, Pointon AD, Cumming BG, Parker AJ (2002) Quantitative analysis of the responses of $\mathrm{V} 1$ neurons to horizontal disparity in dynamic random-dot stereograms. J Neurophysiol 87:191-208.

Robinson DA (1963) A method of measuring eye movement using a scleral search coil in a magnetic field. IEEE Trans Biomed Eng 10:137-145.
Roy JP, Wurtz RH (1990) The role of disparity-sensitive cortical neurons in signalling the direction of self-motion. Nature 348:160-162.

Roy JP, Komatsu H, Wurtz RH (1992) Disparity sensitivity of neurons in monkey extrastriate area MST. J Neurosci 12:2478-2492.

Schlack A, Hoffmann KP, Bremmer F (2002) Interaction of linear vestibular and visual stimulation in the macaque ventral intraparietal area (VIP). Eur J Neurosci 16:1877-1886.

Takahashi K, Gu Y, May PJ, Newlands SD, DeAngelis GC, Angelaki DE (2007) Multimodal coding of three-dimensional rotation and translation in area MSTd: comparison of visual and vestibular selectivity. J Neurosci 27:9742-9756.

Takemura A, Inoue Y, Kawano K (2000) The effect of disparity on the very earliest ocular following responses and the initial neuronal activity in monkey cortical area MST. Neurosci Res 38:93-101.

Takemura A, Inoue Y, Kawano K, Quaia C, Miles FA (2001) Single-unit activity in cortical area MST associated with disparity-vergence eye movements: evidence for population coding. J Neurophysiol 85:2245-2266.

Warren WH (2003) Optic flow. In: The visual neurosciences (Chalupa LM, Werner JS, eds), pp 1247-1259. Cambridge, MA: MIT. 